This article has been accepted for publication in Monthly Notices of the Royal Astronomical Society (C: 2018 The Authors Published by Oxford University Press on behalf of the Royal Astronomical Society. All rights reserved. 


\title{
LOFAR discovery of a double radio halo system in Abell 1758 and radio/X-ray study of the cluster pair
}

\author{
A. Botteon, ${ }^{1,2 \star}$ T. W. Shimwell,,${ }^{3,4}$ A. Bonafede, ${ }^{1,2,5}$ D. Dallacasa, ${ }^{1,2}$ G. Brunetti, ${ }^{2}$ \\ S. Mandal, ${ }^{4}$ R. J. van Weeren, ${ }^{4}$ M. Brüggen, ${ }^{5}$ R. Cassano, ${ }^{2}$ F. de Gasperin, ${ }^{4}$ \\ D. N. Hoang, ${ }^{4}$ M. Hoeft, ${ }^{6}$ H. J. A. Röttgering, ${ }^{4}$ F. Savini, ${ }^{5}$ G. J. White, ${ }^{7,8}$
}

A. Wilber ${ }^{5}$ and T. Venturi ${ }^{2}$

${ }^{1}$ Dipartimento di Fisica e Astronomia, Università di Bologna, via P. Gobetti 93/2, I-40129 Bologna, Italy

${ }^{2}$ INAF - IRA, via P. Gobetti 101, I-40129 Bologna, Italy

${ }^{3}$ ASTRON, the Netherlands Institute for Radio Astronomy, Postbus 2, NL-7990 AA Dwingeloo, the Netherlands

${ }^{4}$ Leiden Observatory, Leiden University, PO Box 9513, NL-2300 RA Leiden, the Netherlands

${ }^{5}$ Hamburger Sternwarte, Universität Hamburg, Gojenbergsweg 112, D-21029 Hamburg, Germany

${ }^{6}$ Thüringer Landessternwarte, Sternwarte 5, D-07778 Tautenburg, Germany

${ }^{7}$ Department of Physical Sciences, The Open University, Milton Keynes MK7 6AA, UK

${ }^{8}$ Space Science and Technology Department, The Rutherford Appleton Laboratory, Chilton, Didcot, Oxfordshire OX11 ONL, UK

\begin{abstract}
Radio haloes and radio relics are diffuse synchrotron sources that extend over Mpc-scales and are found in a number of merger galaxy clusters. They are believed to form as a consequence of the energy that is dissipated by turbulence and shocks in the intracluster medium (ICM). However, the precise physical processes that generate these steep synchrotron spectrum sources are still poorly constrained. We present a new LOFAR observation of the double galaxy cluster Abell 1758. This system is composed of A1758N, a massive cluster hosting a known giant radio halo, and A1758S, which is a less massive cluster whose diffuse radio emission is confirmed here for the first time. Our observations have revealed a radio halo and a candidate radio relic in A1758S, and a suggestion of emission along the bridge connecting the two systems which deserves confirmation. We combined the LOFAR data with archival VLA and GMRT observations to constrain the spectral properties of the diffuse emission. We also analysed a deep archival Chandra observation and used this to provide evidence that $\mathrm{A} 1758 \mathrm{~N}$ and A1758S are in a pre-merger phase. The ICM temperature across the bridge that connects the two systems shows a jump which might indicate the presence of a transversal shock generated in the initial stage of the merger.
\end{abstract}

Key words: radiation mechanisms: non-thermal-radiation mechanisms: thermal-galaxies: clusters: individual: A1758 - galaxies: clusters: intracluster medium-radio continuum: general-X-rays: galaxies: clusters.

\section{INTRODUCTION}

Diffuse and low surface brightness radio emission with steep spectrum $\left(\alpha>1\right.$, with $\left.S_{v} \propto v^{-\alpha}\right)$ associated with the intracluster medium (ICM) has been found in a number of merging galaxy clusters (see Feretti et al. 2012, for an overview). This emission, generally referred to as radio haloes or radio relics, probes the synchrotron radiation from $\sim \mathrm{GeV}$ relativistic electrons in the $\sim \mu \mathrm{G}$ cluster-scale magnetic fields.
Over the last two decades, important steps forward have been made in understanding the origin of non-thermal phenomena in the ICM. A milestone in this framework was the connection between diffuse radio emission in the ICM and dynamically disturbed systems (e.g. Buote 2001; Cassano et al. 2010b, 2013; Cuciti et al. 2015). This suggests that cluster mergers play a crucial role in the formation of haloes and relics. During mergers, turbulence and shocks are generated in the ICM where they dissipate energy. A small fraction of this energy goes into the re-acceleration of relativistic particles and the amplification of the magnetic field (see Brunetti \& Jones 2014, for a review). 
In particular, giant radio haloes are thought to be generated by (primary or secondary) electrons re-accelerated by turbulent-driven mechanisms during mergers (e.g. Brunetti et al. 2001, 2007; Petrosian 2001; Brunetti \& Lazarian 2011, 2016; Brunetti et al. 2017; Pinzke et al. 2017), although many aspects of the physics of these mechanisms remain poorly understood (see Brunetti 2016). Radio relics are thought to be generated by electrons accelerated or reaccelerated by shocks (e.g. Enßlin et al. 1998; Roettiger et al. 1999; Hoeft \& Brüggen 2007; Kang \& Ryu 2011, 2016; Kang et al. 2012; Pinzke et al. 2013). Whilst a connection between radio relics and merger shocks is fairly well established (e.g. Macario et al. 2011; Akamatsu \& Kawahara 2013; Botteon et al. 2016b), open problems include the efficiency of particle acceleration and the proton-toelectron ratio in the acceleration (e.g. Guo et al. 2014a,b; Kang et al. 2014; Vazza \& Brüggen 2014; Vazza et al. 2015a, 2016; Wittor et al. 2017). Shock re-acceleration for radio relics is an emerging scenario that seems to be supported by recent observations of clusters where radio galaxies located close to the relic position can provide seed electrons that are more easily re-accelerated by low Mach number shocks. This alleviates the large requirements of acceleration efficiencies (Bonafede et al. 2014; Shimwell et al. 2015; Botteon et al. 2016a; van Weeren et al. 2017).

The LOw Frequency ARray (LOFAR; van Haarlem et al. 2013) is a radio interferometer observing in the range between 10 and $90 \mathrm{MHz}$ with Low Band Antennas and between 110 and $240 \mathrm{MHz}$ with High Band Antennas (HBA). It has long been recognized that LOFAR has the potential to make breakthroughs in the field of galaxy cluster science (e.g. Röttgering et al. 2006, 2011; Cassano et al. 2010a; Nuza et al. 2012). The high resolution allows for the structures of haloes and relics to be precisely characterized and isolated from the often severe contamination from other radio emitting sources in the vicinity. The dense core provides excellent surface brightness sensitivity, that coupled with the low observing frequencies allows for sensitive observations.

The LOFAR Two-metre Sky Survey (LoTSS; Shimwell et al. 2017) is designed to fully exploit the potential of LOFAR and produce high resolution $(\sim 5 \operatorname{arcsec})$ and high sensitivity $(\sim 100$ $\mu \mathrm{Jy}_{\text {beam }}{ }^{-1}$ ) images of the entire northern sky at $120-168 \mathrm{MHz}$. As part of this survey we have observed the double galaxy cluster Abell 1758. In this paper, we present this new LOFAR observation together with archival Giant Metrewave Radio Telescope (GMRT), Very Large Array (VLA), and Chandra data. Using these data we have discovered and characterized the second double radio halo system known to date and we argue that the two clusters that constitute Abell 1758 are in a pre-merger state.

Throughout this paper, we assume a $\Lambda$ cold dark matter cosmology with $\Omega_{\Lambda}=0.7, \Omega_{\mathrm{m}}=0.3$, and $H_{0}=70 \mathrm{~km} \mathrm{~s}^{-1} \mathrm{Mpc}^{-1}$. At the cluster redshift $(z=0.279)$ this corresponds to a luminosity distance of $D_{\mathrm{L}}=1428 \mathrm{Mpc}$ and to an angular to linear conversion scale of 4.233 arcsec $\mathrm{kpc}^{-1}$. Quoted errors are at $1 \sigma$ confidence level for one parameter, unless otherwise stated.

\section{THE DOUBLE CLUSTER ABELL 1758}

Abell 1758 (hereafter A1758) is a galaxy cluster located at $z=0.279$ that has been intensively studied in the literature. Early ROSAT data (Rizza et al. 1998) revealed that it consists of two components, A1758N (in the north) and A1758S (in the south), separated by a projected distance of $\sim 8$ arcmin (about $2 \mathrm{Mpc}$ ). David \& Kempner (2004) estimated virial radii of $2.6 \mathrm{Mpc}$ (for A1758N) and $2.2 \mathrm{Mpc}$ (for A1758S), indicating that each cluster is affected by the potential well of the other and that they are gravitationally bound.
Despite this, no signs of significant interaction between A1758N and A1758S were found by Chandra and XMM-Newton observations (David \& Kempner 2004). However, from X-ray and optical studies, it is clear that the two sub-clusters are undergoing their own distinct mergers, with $\mathrm{A} 1758 \mathrm{~N}$ in a late and $\mathrm{A} 1758 \mathrm{~S}$ in an early merger state (e.g. David \& Kempner 2004; Boschin et al. 2012). This might also be reflected in the infrared luminosity of the galaxies of A1758N, which is almost two times larger than that of A1758S, suggesting different dynamical histories for the two clusters (Haines et al. 2009). Weak lensing studies indicate that A1758N has a bimodal mass distribution, while A1758S represents a single mass clump (Dahle et al. 2002; Okabe \& Umetsu 2008; Ragozzine et al. 2012; Monteiro-Oliveira et al. 2017). Individual mergers are possibly occurring near the plane of the sky for A1758N and close to the line of sight for A1758S.

So far, most studies have focused on A1758N which is more massive and hotter than A1758S (e.g. David \& Kempner 2004). The mass of A1758N has been estimated using several methods (e.g. X-ray scaling relations, David \& Kempner 2004; weak lensing, Okabe \& Umetsu 2008; member galaxy dynamics, Boschin et al. 2012; hydrostatic equilibrium, Martino et al. 2014), providing a virial mass of $\sim 10^{15} \mathrm{M}_{\odot}$ which is split approximately equally between the two sub-components. This is further supported by hydrodynamical simulations, which can reproduce the X-ray morphology of A1758N assuming an off-axis collision of two equal mass $\left(\sim 5 \times 10^{14} \mathrm{M}_{\odot}\right)$ clusters (Machado et al. 2015). A compilation of the different mass estimates reported for $\mathrm{A} 1758 \mathrm{~N}$ is given in table 1 of Monteiro-Oliveira et al. (2017). Note that the mass of A1758S is more uncertain whilst it appears to be at least a factor of 1.5 smaller than that of A1758N (David \& Kempner 2004; Ragozzine et al. 2012; Haines et al. 2017).

In the radio band, A1758N hosts a giant radio halo that was first detected by Kempner \& Sarazin (2001) and later investigated at $1.4 \mathrm{GHz}$ with the VLA (Giovannini et al. 2009, hereafter G09) and at $325 \mathrm{MHz}$ with the GMRT (Venturi et al. 2013, hereafter V13). There are no reports of diffuse radio emission associated with A1758S in the literature.

\section{OBSERVATIONS AND DATA REDUCTION}

\subsection{LOFAR}

The LoTSS observations are typically separated by $2.6^{\circ}$ and we have analysed the LoTSS pointing that is centred closest to A1758 (offset by $\sim 1.1^{\circ}$ ). The characteristics of this observation are summarized in Table 1. To calibrate the data we followed the facet calibration scheme described in van Weeren et al. (2016b) and Williams et al. (2016). This procedure comprises three steps, which we describe below.

WSCLEAN in the first step, we perform direction-independent calibration (PREFACTOR ${ }^{1}$ pipeline). In this step, the flux calibrator (3C196) data are averaged and bad quality data are flagged. Complex gains and clock offsets for different antenna stations are calibrated off a 3C196 model adopting the absolute flux density scale of Scaife \& Heald (2012). The amplitude and clock solutions are then transferred to the target data before an initial phase calibration against a sky model generated from the TIFR GMRT Sky SurveyAlternative Data Release (TGSS-ADR1; Intema et al. 2017). In the second step, wide field images covering the full LOFAR field

\footnotetext{
${ }^{1}$ https://github.com/lofar-astron/prefactor
} 
Table 1. Summary of the radio observations used in this work.

\begin{tabular}{|c|c|c|c|c|c|}
\hline & \multirow[t]{2}{*}{ LOFAR } & \multirow[t]{2}{*}{ GMRT } & \multicolumn{3}{|c|}{ VLA } \\
\hline & & & Array C & & Array D \\
\hline Project code & LC2_038 & 11TVA01 & & AG639 & \\
\hline Pointing centre (RA, Dec.) & $+49^{\circ} 44^{\prime} 53^{\prime \prime}$ & $+50^{\circ} 30^{\prime} 37^{\prime \prime}$ & & $+50^{\circ} 30^{\prime} 36^{\prime \prime}$ & \\
\hline Observation date & 2014 Jun 1 & 2007 Mar 30/31 & 2004 May 6 & & 2003 Mar 11 \\
\hline Total on-source time (h) & 8.0 & 8.0 & 2.5 & & 2.5 \\
\hline Central frequency $(\mathrm{MHz})$ & 144 & 325 & & 1425 & \\
\hline Bandwidth (MHz) & 48 & 33 & & 50 & \\
\hline
\end{tabular}

of view (FoV) are made from the products of the first step using (Offringa et al. 2014). The PYthon Blob Detector and Source Finder (PYBDSF; Mohan \& Rafferty 2015) software is then used to detect sources which are then subtracted from the $u v$-data using the clean component models. Images are produced at medium $(\sim 40$ $\operatorname{arcsec} \times 30 \operatorname{arcsec})$ and low resolution $(\sim 120 \operatorname{arcsec} \times 100 \operatorname{arcsec})$ to ensure that both compact and extended sources are subtracted. The image sizes are about $12^{\circ} \times 12^{\circ}$ and $30^{\circ} \times 30^{\circ}$ for medium and low resolution, respectively.

In the direction-dependent calibration step (FACTOR ${ }^{2}$ pipeline), nearly thermal noise limited images can be produced. It operates by using bright sources in the field to calibrate the phases and amplitudes in a restricted portion of the sky, i.e. a 'facet'. This is needed as the LOFAR primary beam is large, requiring many different corrections for ionospheric distortions and beam model errors across the FoV. For this reason, the FoV (usually a region within $2.5^{\circ}$ from the pointing centre) is divided into tens of facets that are typically processed in order of decreasing flux density of the calibrator source. After a number of self-calibration cycles on the facet calibrator, the fainter sources that were subtracted in the second step are added back into the data and are calibrated using the solutions derived from the facet calibrator. An updated sky model of the region covered by the facet is obtained by subtracting the components of the processed facet from the $u v$-data. The $u v$-data that is then used for the calibration of the subsequent facet has smaller systematics due to the source subtraction and the effective noise in the data set is reduced. This process is iteratively repeated until all the desired directions have been calibrated. This procedure has been successfully applied to image several other galaxy clusters with the LOFAR HBA (e.g. van Weeren et al. 2016a; de Gasperin et al. 2017; Hoang et al. 2017; Wilber et al. 2018).

All LOFAR images in the paper are reported at the central observing frequency of $144 \mathrm{MHz}$ and were produced with CASA v4.7 (McMullin et al. 2007). The imaging was done using the multiscale multifrequency deconvolution algorithm (Rau \& Cornwell 2011), with second order Taylor terms (nterms $=2$ ), and W-projection (Cornwell et al. 2005). For the facet containing A1758, an image size of $5120 \operatorname{arcsec} \times 5120$ arcsec was adopted to ensure that all of the sources in the vicinity of the cluster were adequately deconvolved. An inner $u v$-cut of $80 \lambda$ (corresponding to an angular scale of $43 \mathrm{arcmin}$ ) was also applied on the data to reduce sensitivity to very large scale emission from the Galaxy. Different resolution images were created using various different Briggs weightings (Briggs 1995 ) and by applying an $u v$-taper, as reported in Table 2 . Primary beam correction was performed with AWIMAGER (Tasse et al. 2013).

\footnotetext{
${ }^{2}$ https://github.com/lofar-astron/factor
}

Uncertainties in the flux scale that are caused by inaccuracies in the LOFAR HBA beam model (see Hardcastle et al. 2016; van Weeren et al. 2016b) were corrected by cross-matching a number of compact sources extracted from the LOFAR image with the TGSS (Intema et al. 2017). Throughout the paper we have applied correction factor that was computed from the mean LOFAR/TGSS integrated flux density ratio of 1.08 and a calibration error of 15 per cent on LOFAR flux densities, which is in agreement with other LOFAR HBA studies (e.g. Shimwell et al. 2016; Savini et al. 2018)

\subsection{GMRT}

We analysed an $8 \mathrm{~h}$ archival GMRT $325 \mathrm{MHz}$ observation of A1758 (details in Table 1). Data were reduced with the Source Peeling and Atmospheric Modelling (SPAM) package (Intema et al. 2009), which is an automated pipeline to process GMRT observations based on the Astronomical Image Processing System (AIPS). Here we outline the main steps of the SPAM data reduction, for more details the reader is referred to Intema et al. (2009, 2017). First, the data set is averaged in time and frequency to reduce the data processing time whilst keeping enough resolution in both time and frequency to avoid smearing. Bad data due to corrupted baselines, non-working antennas, and radio frequency interference were also excised. The bandpass and absolute flux density scale were calibrated using the primary flux calibrator 3C147 and adopting the Scaife \& Heald (2012) flux scale. An initial phase-only calibration using a sky model generated from the VLA Low-frequency Sky Survey Redux (VLSSr; Lane et al. 2014), the WEsterbork Northern Sky Survey (WENSS; Rengelink et al. 1997), and the NRAO VLA Sky Survey (NVSS; Condon et al. 1998) was followed by a number of loops of self-calibration, wide-field imaging, and additional flagging of bad data. Then the bright sources in the primary beam are used to perform a direction-dependent calibration and ionospheric modelling aiming to mitigate the phase errors introduced by the ionosphere. The final calibrated data were then imaged with CASA v4.7, in the manner that was described at the end of Section 3.1. In our analysis we did not consider the effect of variation in system temperature (see Sirothia 2009). Instead, we adopted a similar approach to that described in Section 3.1, cross-matching a number of sources extracted from the GMRT image with the WENSS (Rengelink et al. 1997) and applying a correction factor of 0.73 on the GMRT flux densities with a systematic uncertainty of 15 per cent (see Chandra et al. 2004).

\subsection{VLA}

We analysed archival VLA observations of A1758 at $1.4 \mathrm{GHz}$ in configurations $\mathrm{C}$ and $\mathrm{D}$. The details of the observations are reported 
Table 2. Imaging parameters for the radio images shown in the paper. The beam position angle (PA) is measured from north to east.

\begin{tabular}{lccccccc}
\hline Fig. & Instrument & $\begin{array}{c}\text { Frequency } \\
(\mathrm{MHz})\end{array}$ & Robust & $\begin{array}{c}\text { Taper } \\
(\operatorname{arcsec})\end{array}$ & $\begin{array}{c}\text { Resolution } \\
(\operatorname{arcsec} \times \operatorname{arcsec})\end{array}$ & $\begin{array}{c}\text { PA } \\
\left(^{\circ}\right)\end{array}$ \\
\hline 1 & LOFAR & 144 & +0.5 & 0 & $34 \times 23$ & 45 \\
$\left(\mu \mathrm{Jybeam}{ }^{-1}\right)$
\end{tabular}

in Table 1. Data reduction was performed with AIPS where the two data sets were edited, calibrated, and imaged separately. Thus the $u v$-data were combined to produce a single image of the cluster. The flux calibrator of VLA observations was 3C286 (model from Perley \& Butler 2013). The final imaging was performed with CASA v4.7 as described in Section 3.1. The absolute flux scale calibration errors were conservatively set to 5 per cent on VLA flux densities.

\subsection{Integrated synchrotron spectra and source subtraction}

Given the different $u v$-coverage of the LOFAR, GMRT, and VLA observations, it was necessary to match the $u v$-sample of the different interferometers as closely as possible to provide an accurate comparison between the flux densities measured at different frequencies, and to compute the diffuse emission spectra. As a first step, we removed the discrete sources from each data sets. This procedure was performed by applying an inner $u v$-cut of $2.0 \mathrm{k} \lambda$ (corresponding to an angular scale of $103 \mathrm{arcsec}$, i.e. about $440 \mathrm{kpc}$ at $z=0.279$ ) to the data to image only the compact sources in the field whose clean components were subsequently subtracted from the visibilities. To image the diffuse emission after the source subtraction, for each data set we used an inner $u v$-cut of $170 \lambda$ and uniform weighting. A Gaussian $u v$-taper of 35 arcsec was also used to enhance diffuse emission and to produce images with comparable beams. Errors on flux densities were estimated via

$\Delta S=\sqrt{\left(\sigma_{\mathrm{rms}} \times \sqrt{\frac{A_{\mathrm{s}}}{A_{\mathrm{b}}}}\right)^{2}+(\xi \times S)^{2}}$,

where $\xi$ is the calibration uncertainty, $\sigma_{\text {rms }}$ is the root-mean-square noise while $A_{\mathrm{s}}$ and $A_{\mathrm{b}}$ are the source and beam areas, respectively.

In Fig. 1 we present the LOFAR image (left-hand panel) along with the point-source-subtracted images from the GMRT (central panel) and VLA (right-hand panel). This choice has been made since the diffuse emission is best visible.

\subsection{Chandra}

We retrieved three ACIS-I observations (ObsID: 13997, 15538, 15540) on A1758 from the Chandra data archive ${ }^{3}$ for a total exposure time of $150 \mathrm{ks}$. We mention that two other Chandra pointings on A1758 also exist; however they composed of an ACIS-S observation where only A1758N is in the FoV (ObsID 2213) and by a short (7 ks) observation (ObsID: 7710) whose exposure time is negligible with respect to the total integration time. For that reason they were not used in this work.

Data reduction was performed with CIAO v 4.9 and Chandra CALDB v4.7.3. Time periods affected by soft proton flares were removed

${ }^{3}$ http://cda.harvard.edu/chaser/ by inspecting the light curves in the $0.5-7.0 \mathrm{keV}$ band extracted for each ObsID from the S2 chip with the 1C_clean routine. After this step, the resulting clean exposure time is $137 \mathrm{ks}$. We used merge_obs to add together the three data sets and produce the final cluster image in the $0.5-2.0 \mathrm{keVband}$ shown in Fig. 2. An exposurecorrected point spread function (PSF) map with minimum size was created from the combination of the PSF and exposure maps of the three ObsIDs. This was used to detect discrete sources with the wavdetect task, which were later confirmed by eye and excluded in the further analysis.

Spectra were extracted in the same regions from all the ObsIDs and simultaneously fitted in the $0.5-10.0 \mathrm{keV}$ band with XSPEC v12.9.0o (Arnaud 1996). The background was carefully treated with a model that included both astrophysical and instrumental emission components, as shown in Fig. 3. The former is described by two main components due to: the Galactic emission, modelled with two thermal plasmas with $k T_{1}=0.14 \mathrm{keV}$ (unabsorbed) and $k T_{2}=0.25 \mathrm{keV}$ (absorbed), and the cosmic X-ray background, described with an absorbed power law with photon index $\Gamma=1.4$. For the latter we followed Bartalucci et al. (2014) which provided an analytical model for the ACIS-I particle background. Spectra were fitted adopting Cash statistics (Cash 1979) and an absorbed thermal model for the ICM with metallicity fixed at $0.3 \mathrm{Z} \odot$ (solar abundance table by Anders \& Grevesse 1989) and hydrogen column density $N_{\mathrm{H}}=1.03 \times 10^{20} \mathrm{~cm}^{-2}$ computed from the Leiden/Argentine/Bonn Survey of Galactic H I (Kalberla et al. 2005).

We used contbin v1.4 (Sanders 2006) to compute the thermodynamical properties of the ICM in A1758. A signal-to-noise ratio $(\mathrm{S} / \mathrm{N})$ of 50 for the net counts in the $0.5-2.0 \mathrm{keV}$ band was set to delineate the regions where spectra were extracted and fitted as written above. For more details on the computation of the maps of the ICM thermodynamical quantities and generally on the Chandra data analysis, we refer the reader to Botteon et al. (2018) in which the same procedures adopted in the current paper have been more thoroughly described.

\section{RESULTS}

\subsection{A1758N radio halo}

Diffuse emission in A1758N is visible both from the NVSS and the WENSS surveys (Kempner \& Sarazin 2001). The observations taken with the VLA at $1.4 \mathrm{GHz}$ (G09) and with the GMRT at $325 \mathrm{MHz}$ (V13) confirmed the presence of a giant radio halo which is elongated in the NW-SE direction and only partially covering the $\mathrm{X}$-ray emission of the cluster. The spectral index reported between these two frequencies is $\alpha=1.31 \pm 0.16$ (V13).

LOFAR detects the extended radio halo flux in A1758N at higher significance and the recovered morphology appears consistent with the GMRT and VLA maps, as demonstrated in Fig. 1. We measure a largest linear size of the emission of $\sim 2.2 \mathrm{Mpc}$. The low-resolution 

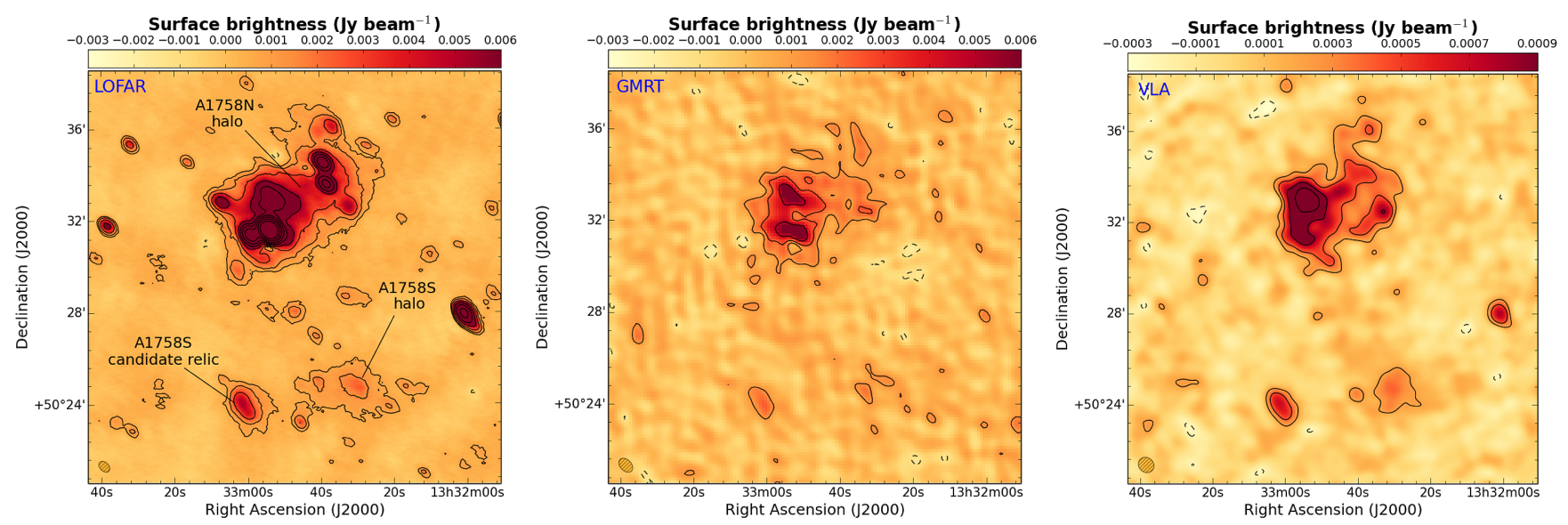

Figure 1. The cluster A1758 as observed in the radio band with LOFAR (left), GMRT (centre), and VLA (right). The point sources were subtracted in the GMRT and VLA images. Contours are spaced by a factor of 2 starting from $3 \sigma$, where $\sigma_{\mathrm{LOFAR}}=230 \mu \mathrm{Jy}_{\text {beam }}^{-1}, \sigma_{\mathrm{GMRT}}=400 \mu \mathrm{Jy}$ beam ${ }^{-1}$, and $\sigma_{\mathrm{VLA}}=70$ $\mu \mathrm{Jy}$ beam $^{-1}$. The negative $-3 \sigma$ contours are shown in dashed. The beam sizes are $34 \operatorname{arcsec} \times 23 \operatorname{arcsec}($ LOFAR), $43 \operatorname{arcsec} \times 29 \operatorname{arcsec}(\mathrm{GMRT})$, and 43 $\operatorname{arcsec} \times 38 \operatorname{arcsec}(\mathrm{VLA})$ and are shown in the bottom left corners. More details on the images are reported in Table 2.

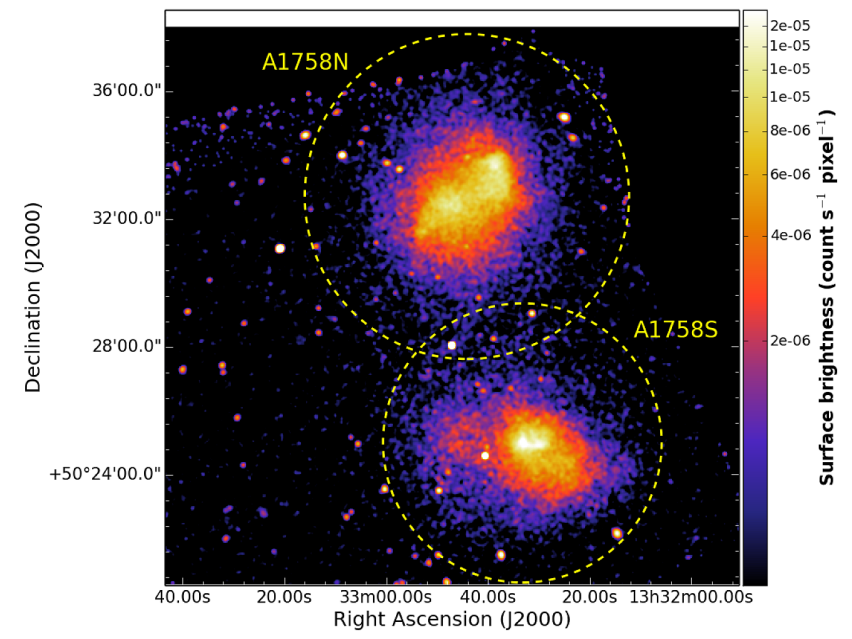

Figure 2. Chandra exposure-corrected image in the $0.5-2.0 \mathrm{keV}$ band of A1758 smoothed to a resolution of $\sim 3$ arcsec. Yellow circles indicate the approximate location of $r_{500}$ for each cluster.

point-source-subtracted LOFAR contours displayed in Fig. 4 suggest that the non-thermal radio emission in A1758N covers the X-ray bright region of the cluster. At higher resolution (Fig. 5, left-hand panel), only the brightest part of the radio halo is visible; in particular, LOFAR shows two bright and straight structures (labelled as S1 and S2 in Fig. 5, left-hand panel) apparently not associated with any optical galaxy. They might indicate regions where the plasma has been somehow locally compressed or re-accelerated (e.g. Shimwell et al. 2016; de Gasperin et al. 2017). The feature S1 is also detected with the GMRT and VLA (Fig. 1). In the southeast, A1758N also hosts two prominent narrow angle tailed radio galaxies labelled as A and B in Fig. 5 (left-hand panel). The former (also identified as $1330+507$ ) was studied at high resolution with the VLA by O'Dea \& Owen (1985).

The presence of resolved radio galaxies embedded in the halo (e.g. source A and B in Fig. 5, left-hand panel) makes it difficult to disentangle their contribution from that of the halo. We repeated the subtraction by adopting inner $u v$-cuts in the range $1.0-3.5 \mathrm{k} \lambda$,

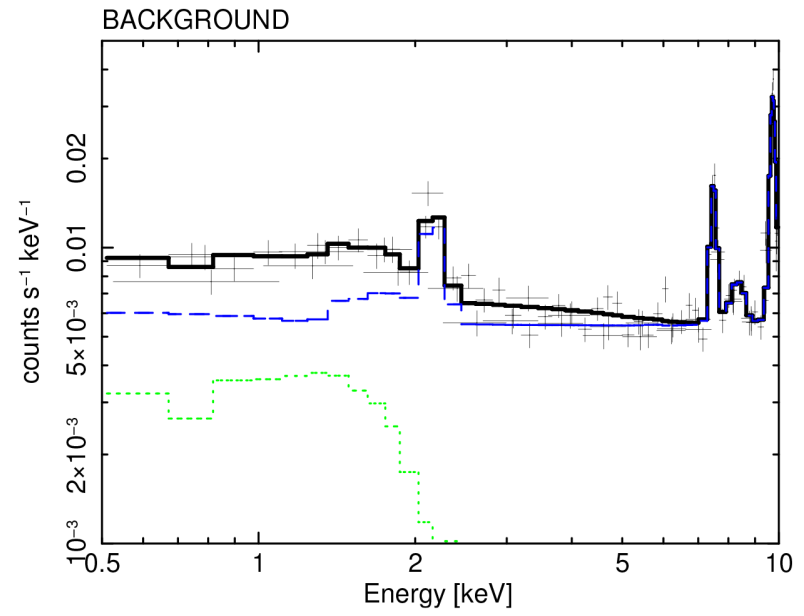

Figure 3. Spectrum of the Chandra background. Data points are shown in black together with the best-fitting model. The astrophysical and instrumental backgrounds are shown in dotted green and dashed blue, respectively. Whilst the three ObsID spectra were simultaneously fitted, the models for only one observation were reported in order to avoid confusion in the plot. The c-stat/d.o.f. of the fit is $406 / 386$.

corresponding to linear sizes of $873-249 \mathrm{kpc}$ at the cluster redshift, to assess the uncertainties in our source subtraction on the LOFAR data set, in addition to the procedure described in Section 3.4. In Fig. 6 we show how the flux density measurement of the northern radio halo varies with the $u v$-cut, ranging from 415 to $483 \mathrm{mJy}$ (the mean value is $440 \mathrm{mJy}$ ). This indicates that the choice of the $u v$-cut has an impact on the halo in A1758N. In contrast, the integrated flux density of the diffuse sources in A1758S (see sections below) is essentially independent on the $u v$-cut used (Fig. 6), indicating that the subtraction is less problematic which is expected as there are just a few weak discrete sources without significant extended emission (cf. Fig. 5, right-hand panel).

In Table 3 and Fig. 7 we report the flux density measurements at three frequencies and the spectra, respectively, of the diffuse emission in A1758. The spectral index between $144 \mathrm{MHz}$ and $1.4 \mathrm{GHz}$ is $\alpha=1.2 \pm 0.1$ for the halo in $\mathrm{A} 1758 \mathrm{~N}$, consistent with that of 


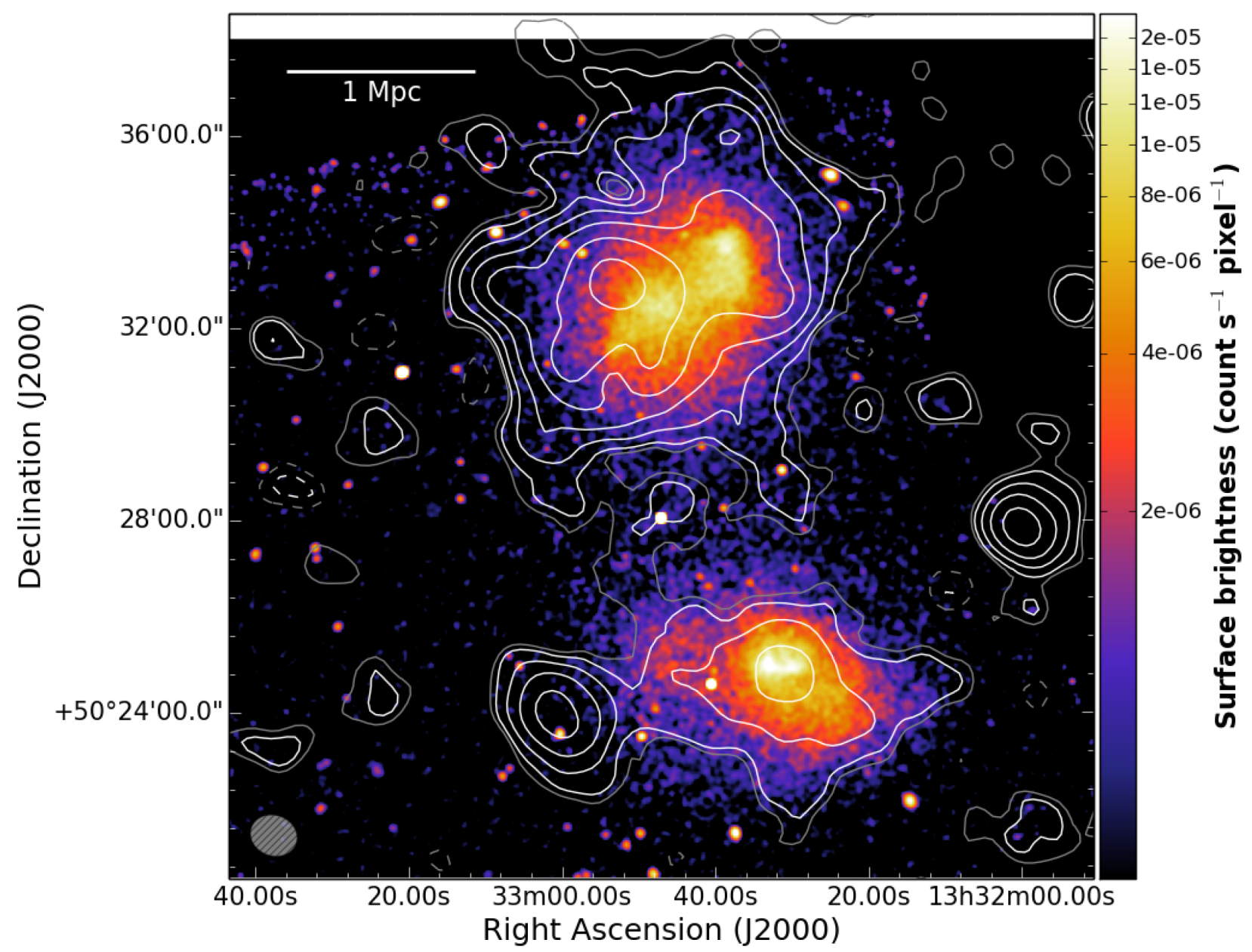

Figure 4. LOFAR radio contours with point sources subtracted of A1758 overlaid on the Chandra colour image of Fig. 2. The LOFAR white contours are spaced by a factor of 2 starting from $3 \sigma$, where $\sigma_{\mathrm{LOFAR}}=390 \mu \mathrm{Jy}$ beam $^{-1}$. The negative $-3 \sigma$ contours are shown in dashed. Grey contours correspond to the $\pm 2 \sigma$ level. The beam size is $60 \operatorname{arcsec} \times 51$ arcsec and is shown in the bottom left corner. More details on the LOFAR image are reported in Table 2 .
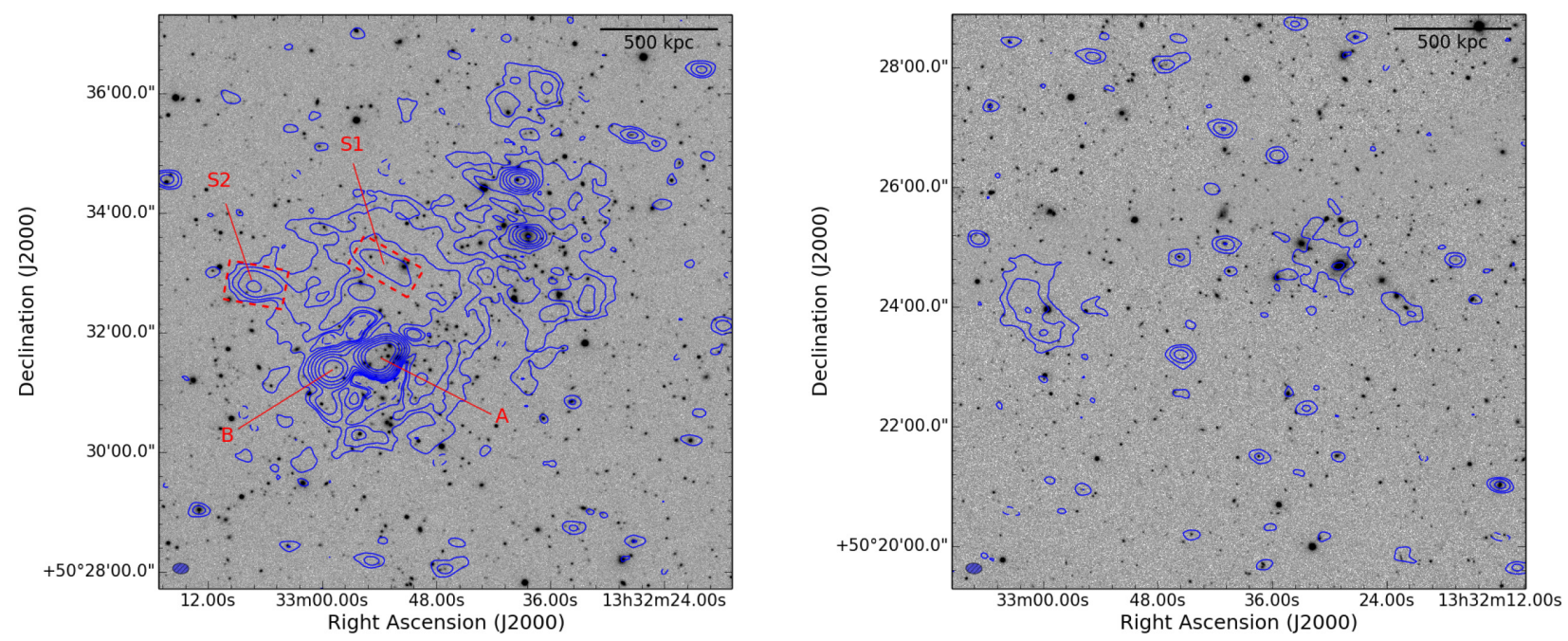

Figure 5. Mosaic SDSSg,r,i images of A1758N (left) and A1758S (right) overlaid with the LOFAR contours spaced by a factor of 2 starting from $3 \sigma$, where $\sigma_{\text {LOFAR }}=140 \mu \mathrm{Jy}_{\text {beam }}{ }^{-1}$. The negative $-3 \sigma$ contours are shown in dashed. The beam size is $16 \operatorname{arcsec} \times 11$ arcsec and is shown in the bottom left corners. At this resolution the radio halo in A1758S is marginally visible. More details on the LOFAR image are reported in Table 2.

$\alpha=1.31 \pm 0.16$ computed by V13. The flux densities measured in our GMRT and VLA images agree to that reported in V13 within
$1 \sigma$. The emission from the potential relic and the halo in A1758S have not been previously reported. 


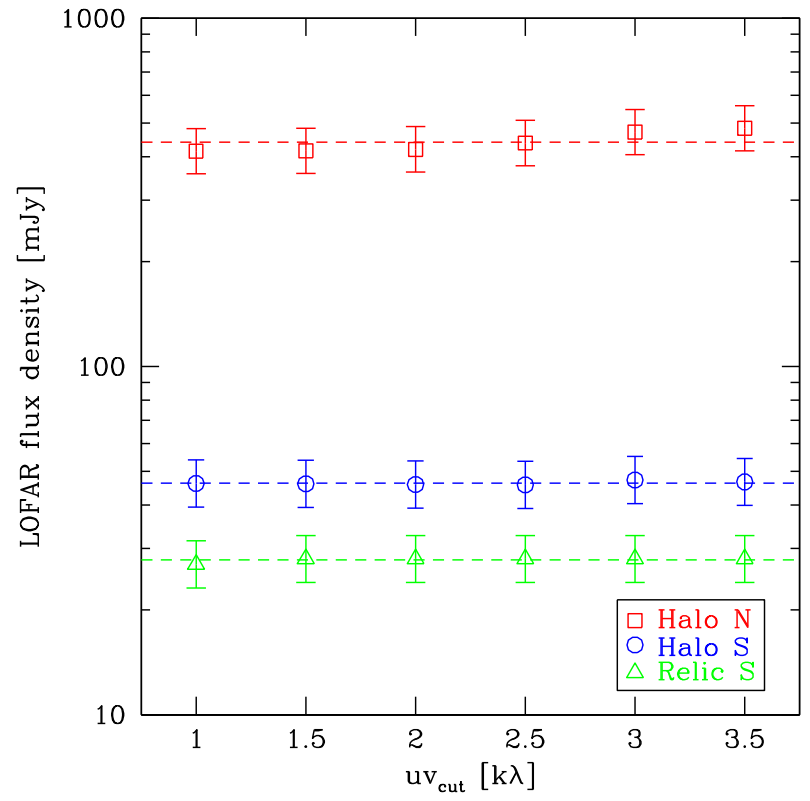

Figure 6. The flux densities of the diffuse emission in A1758 measured with LOFAR versus the inner $u v$-cuts adopted to subtract the point sources. Dashed horizontal lines show the mean values of the measurements.

Table 3. Flux densities of the diffuse emission in A1758. The spectral indexes were computed adopting the procedure described in Section 3.4.

\begin{tabular}{lccr}
\hline$v(\mathrm{MHz})$ & Halo N & $\begin{array}{c}S_{v}(\mathrm{mJy}) \\
\text { Halo S }\end{array}$ & \multicolumn{1}{c}{ Relic S } \\
\hline 144 & $420 \pm 63$ & $45.8 \pm 7.1$ & $28.0 \pm 4.3$ \\
325 & $134 \pm 20$ & $16.8 \pm 3.5$ & $8.9 \pm 2.0$ \\
1425 & $24.7 \pm 1.7^{a}$ & $3.1 \pm 0.7^{a}$ & $1.5 \pm 0.3$ \\
$\alpha$ & $1.2 \pm 0.1$ & $1.1 \pm 0.1$ & $1.3 \pm 0.1$ \\
\hline
\end{tabular}

$\overline{{ }^{a}}$ The error takes into account also the uncertainties of the source subtraction.

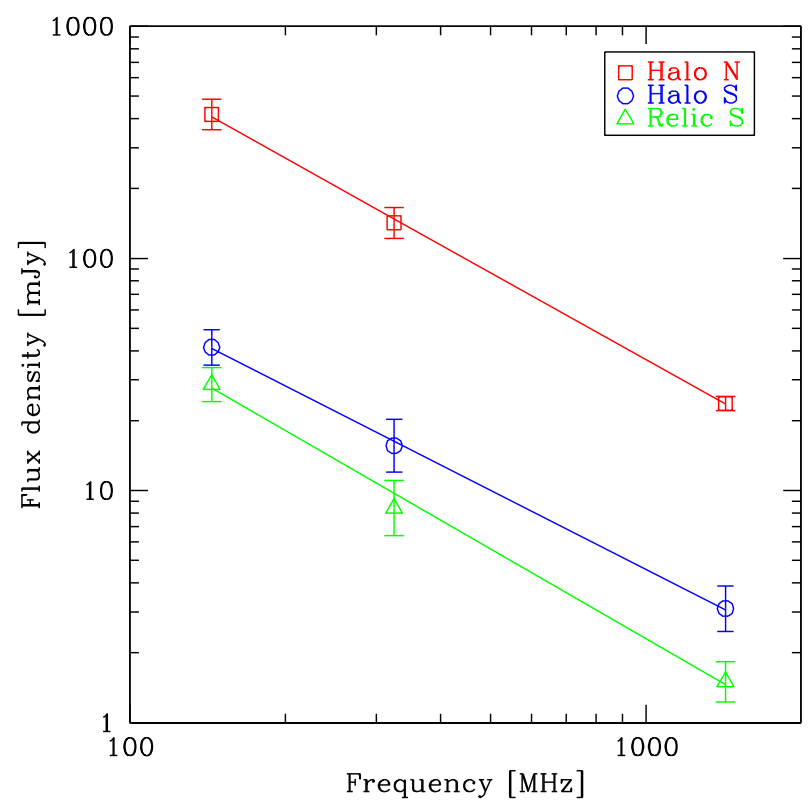

Figure 7. Integrated spectra of the diffuse radio emissions in A1758. The spectral index values are reported in Table 3.

\subsection{A1758S radio halo}

The halo in A1758S is barely visible with the GMRT and it is detected at low significance with the VLA (Fig. 1). The characteristics of the emission recovered by the LOFAR observation (Fig. 4) are typical for a radio halo, i.e. low surface brightness, similar morphology with respect to the ICM thermal emission and a largest linear size of $\sim 1.6 \mathrm{Mpc}$. It is worth noting that the merger axis of A1758S is likely close to the line of sight (e.g. Monteiro-Oliveira et al. 2017), hence we cannot fully discard the possibility that the radio emission traces a radio relic observed face-on, although this is unlikely because the remarkable similarity between the radio and the X-ray emission (Fig. 4). Future studies on the source polarization level will definitely clarify this point.

The integrated flux density measured with LOFAR within the $3 \sigma$ contour from the low-resolution image of Fig. 4 (excluding the peripheral emission to the east, see section below) is $45.8 \pm 7.1 \mathrm{mJy}$, i.e. one order of magnitude lower than that of the halo in A1758N. Indication of the presence of a radio halo in A1758S has also been found in the Westerbork Synthesis Radio Telescope (WSRT) data at $367 \mathrm{MHz}$ (Drabent 2017). However, the available observation is not suitable to study in detail the diffuse emission due to its inadequate angular resolution which makes the point-source subtraction unreliable. The spectral index computed between $144 \mathrm{MHz}$ and $1.4 \mathrm{GHz}$ within a region traced by the LOFAR emission is $\alpha=1.1 \pm 0.1$ (see Table 3) and the fit is shown in Fig. 7. We also determined the halo spectral index considering a region defined by the VLA $3 \sigma$ contour. In this case, the flux densities evaluated in the LOFAR and VLA images are $\sim 12.5$ and $\sim 1.2 \mathrm{mJy}$, respectively, and the spectral index is consistent with that reported above.

\subsection{A1758S candidate radio relic}

To the east of A1758S, an extended radio source at the boundaries of the X-ray emission is observed with LOFAR, GMRT, and VLA (Fig. 1). In the LOFAR low-resolution contours of Fig. 4 , the $3 \sigma$ contour of this emission is connected with that of the radio halo. In Table 3 we report the flux density measurements at various frequencies. We estimated a spectral index between $144 \mathrm{MHz}$ and $1.4 \mathrm{GHz}$ of $\alpha=1.3 \pm 0.1$ for this source (Fig. 7). We tentatively classify this emission as a radio relic based on the following characteristics: (i) its elongated morphology roughly arc-shaped and perpendicular to the thermal cluster emission, (ii) its largest linear size $>500 \mathrm{kpc}$, (iii) its peripheral location in the same direction of the ICM elongation, (iv) its steep spectrum, and (v) the absence of a clear optical counterpart ${ }^{4}$ and/or bright compact radio emission (Fig. 5, right-hand panel). All these properties are commonly observed in radio relics but can also be seen in other objects, such as dead radio galaxies (e.g. Brienza et al. 2016, and references therein). A definitive claim would require either the study of the spectral index gradient towards the cluster centre, measurements of the source polarization or the detection in the X-rays of an underlying shock front. Unfortunately none of these measurements can be carried out with the present data.

\footnotetext{
${ }^{4}$ Note that the source roughly at the centre of the diffuse radio emission in the optical image of Fig. 5 (right-hand panel) is a star. No redshift has been reported for the X-ray point source (identified as SDSS J133300.32+502332.2) embedded in the candidate relic that is visible in the Chandra image of Fig. 4. With the current data we cannot conclude whether it is associated with the radio emission.
} 


\subsection{X-ray properties of A1758N and A1758S}

The deep Chandra observation of A1758 allowed us to derive the projected maps of the ICM thermodynamical quantities shown in Fig. 8. The temperature map displays overall higher values in A1758N than in A1758S. These values are within the range of $8.0-9.9 \mathrm{keV}$, for $\mathrm{A} 1758 \mathrm{~N}$, and $6.0-6.7 \mathrm{keV}$, for A1758S, that were reported by David \& Kempner (2004) who made measurements within $1 \mathrm{Mpc}$ radius aperture centred on the centroid of each cluster. Shock heated regions towards the NW and SE of the northern cluster are suggested by high values of temperature and pressure (Fig. 8, see also Fig. A2), in agreement with the late merger scenario (David \& Kempner 2004) where the shocks have already crossed the central region of the ICM and are moving outwards with high Mach numbers (Machado et al. 2015). Our entropy map of Fig. 8 (righthand panel) highlights the presence of the two cores in $\mathrm{A} 1758 \mathrm{~N}$ and the single core in A1758S, characterized by the lowest values of entropy in the map, in line with the bimodal (A1758N) and single clump (A1758S) mass distribution already inferred from optical studies (Dahle et al. 2002; Okabe \& Umetsu 2008; Ragozzine et al. 2012; Monteiro-Oliveira et al. 2017).

\subsection{The bridge between A1758N and A1758S}

The maps of the ICM thermodynamical quantities shown in Fig. 8 show a complex thermodynamics in the region between $\mathrm{A} 1758 \mathrm{~N}$ and A1758S, suggesting that the two clusters are in early interaction. At this stage of the interaction the gas between them is compressed and heated, somewhat explaining the observed high values of temperature $(\sim 7.5 \mathrm{keV})$ and pressure in the region between the cluster pair. Moreover, the entropy map (Fig. 8, right-hand panel) displays the largest values in such a region, further indicating an unrelaxed state of the clusters outskirts.

The LOFAR low-resolution contours of Fig. 4 give a tantalizing hint of a low surface brightness bridge connecting A1758N and A1758S. This emission is detected at the $2 \sigma$ level towards the eastern edge of the region between the two clusters. On the western edge of this region, a protuberance of the A1758N halo extends towards A1758S. Although particular care was devoted in the subtraction of the point sources between the clusters, the blending of low-level residual emission due to faint and unresolved sources, combined with the large synthesized beam of the image and with the non-uniform distribution of the noise, could mimic the filamentary structure. All this, together with the low significance level of the emission, does not allow us to make a firm statement about its presence. Nonetheless, filaments connecting galaxy clusters are expected to be observed in the radio band even on larger scales (e.g. Keshet et al. 2004; Araya-Melo et al. 2012; Vazza et al. 2015b). We note that a hint of a Sunyaev-Zel'dovich (SZ) signal connecting A1758N and A1758S was reported also in AMI Consortium (2012).

We analysed the thermal properties of the X-ray emission between A1758N and A1758S, visible by smoothing to a resolution of $\sim 15$ arcsec the Chandra $0.5-2.0 \mathrm{keV}$ image (Fig. 9, left-hand panel). We extracted spectra from five regions enclosing $\sim 1000$ counts each in the $0.5-2.0 \mathrm{keV}$ band that were fitted as described in Section 3.5. The best-fitting spectra are reported in Appendix B. These were used to compute the temperature profile shown in Fig. 9 (right-hand panel). We measure high $k T$ values inside the $\mathrm{X}$-ray channel that drop by a factor of $\sim 3$ between regions 2 and 1 (see Appendix A for the apparent discrepancy between the values of the temperature map and profile shown in Fig. 9); if projection effects play a role, the temperature drop would be even larger. Unfortunately, the count statistics does not allow us to increase spatial resolution to firmly understand if this is sharp drop or a gradual decrement. If we assume that this is a jump due to a shock and we apply the Rankine-Hugoniot jump conditions (e.g. Landau \& Lifshitz 1959)

$\frac{T_{2}}{T_{1}}=\frac{5 \mathcal{M}_{\mathrm{kT}}^{4}+14 \mathcal{M}_{\mathrm{kT}}^{2}-3}{16 \mathcal{M}_{\mathrm{kT}}^{2}}$

the derived Mach number ${ }^{5}$ would be $\mathcal{M}_{\mathrm{kT}}=3.0_{-1.0}^{+1.4}$. We notice that this putative shock is co-located with the $2 \sigma$ level emission observed by LOFAR (Fig. 9, inset in the left-hand panel). This is tantalizing and deserves future follow-ups as it might suggest a connection between the shock and the possible radio bridge. Its uncommonly high Mach number and unusual transversal location are in agreement with the recent work of Ha et al. (2018), where these kind of shocks are referred as 'equatorial'. Alternatively, the high $k T$ values of the X-ray channel could be due to the adiabatic compression of the gas in the filament connecting A1758N and A1758S during the initial stage of the merger.

As a complementary information, the five spectra were used to compute the profiles of emission measure, pressure, and entropy shown in Fig. 9 (right-hand panel). These quantities are also observed to jump from the external to internal regions. We urge caution when interpreting these measurements as they were derived from the normalization of the cluster thermal model, that is $\propto n^{2} L$, and are projected along the line of sight $L$ ( $n$ is the density of the medium). For this reason they are usually referred as 'pseudo' quantities (e.g. Mazzotta et al. 2004). We can assess the effects of projection assuming a cylindrical shape for the X-ray channel (Fig. 9, left-hand panel) and using the dependencies on the line of sight of the emission measure $\left(\propto L^{-1}\right)$, pressure ( $\propto L^{-1 / 2}$ ) and entropy ( $\left.\propto L^{1 / 3}\right)$. For example, the ratio between the quantities measured at centre and $r_{5}\left(\equiv r_{1}\right)$, i.e. the distance of the outermost region, changes by $<16$ per cent (or $<34$ per cent) for emission measure, $<8$ per cent (or $<16$ per cent) for pressure and $<4$ per cent (or $<10$ per cent) for entropy for cylinder radii $R>2 r_{5}$ (or $>1.5 r_{5}$ ).

\section{DISCUSSION}

A1758 is an ideal object to study the merger processes between galaxy clusters and the impact of these events on their environment. Indeed, this system is composed of two main components, A1758N and A1758S, in different evolutionary stages (see Section 2).

The diffuse radio emission in A1758 follows the X-ray emission of the ICM (Fig. 4), suggesting a relation between the thermal and non-thermal components. The double cluster A1758N and A1758S represents the second system known to date to host two radio haloes. The first discovered is the pair A399-A401 (Murgia et al. 2010), located at $z=0.07$. Both A1758 and A399-A401 show the presence of an X-ray channel between the cluster pair and some evidence for a lateral shock (Akamatsu et al. 2017). The bridge connecting A399 and A401 is more clear and indeed it has been also observed via the SZ effect by the Planck satellite (Planck Collaboration VIII 2013);

\footnotetext{
${ }^{5}$ Although also the surface brightness drops outside the X-ray channel, we did not attempt the 'canonical' broken power-law density profile fit (e.g. Markevitch \& Vikhlinin 2007) to search for the edge due to the complex geometrical problem at this location (overlap of the outskirts of two galaxy clusters).
} 

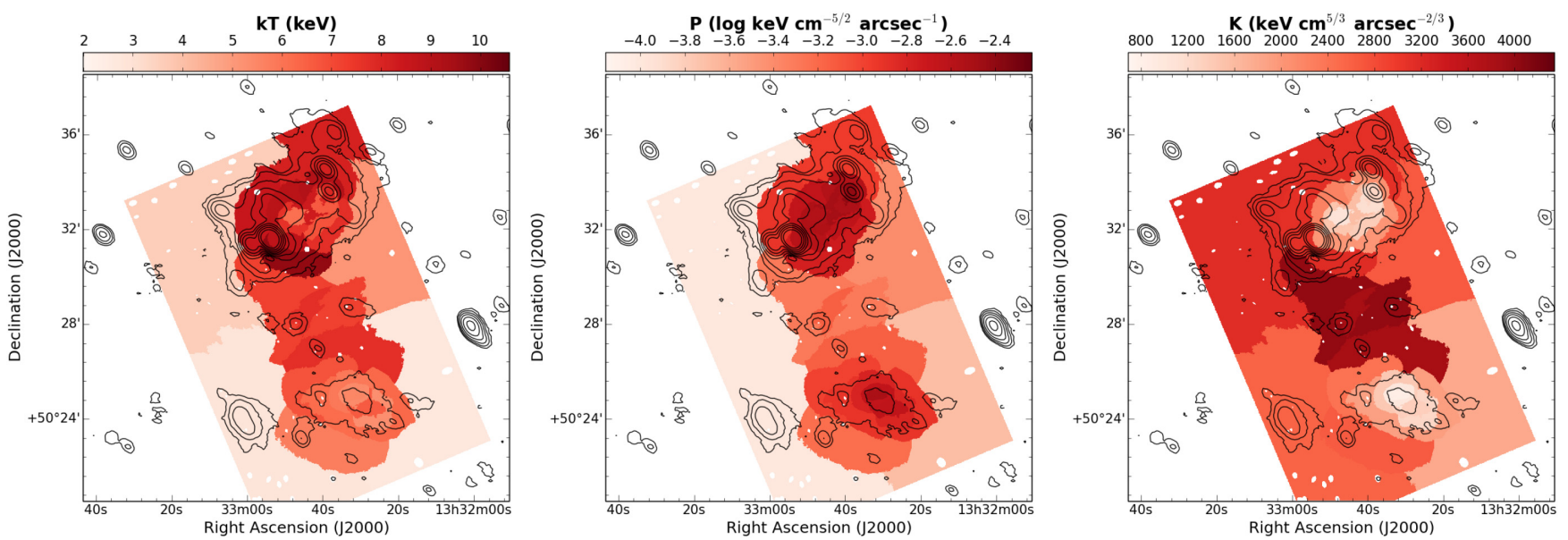

Figure 8. Thermodynamical properties of the ICM in A1758 with overlaid the LOFAR contours of Fig. 1. Images depict projected values of temperature (left), pressure (centre), and entropy (right). The temperature error map and a lower S/N temperature map are reported in Appendix A.
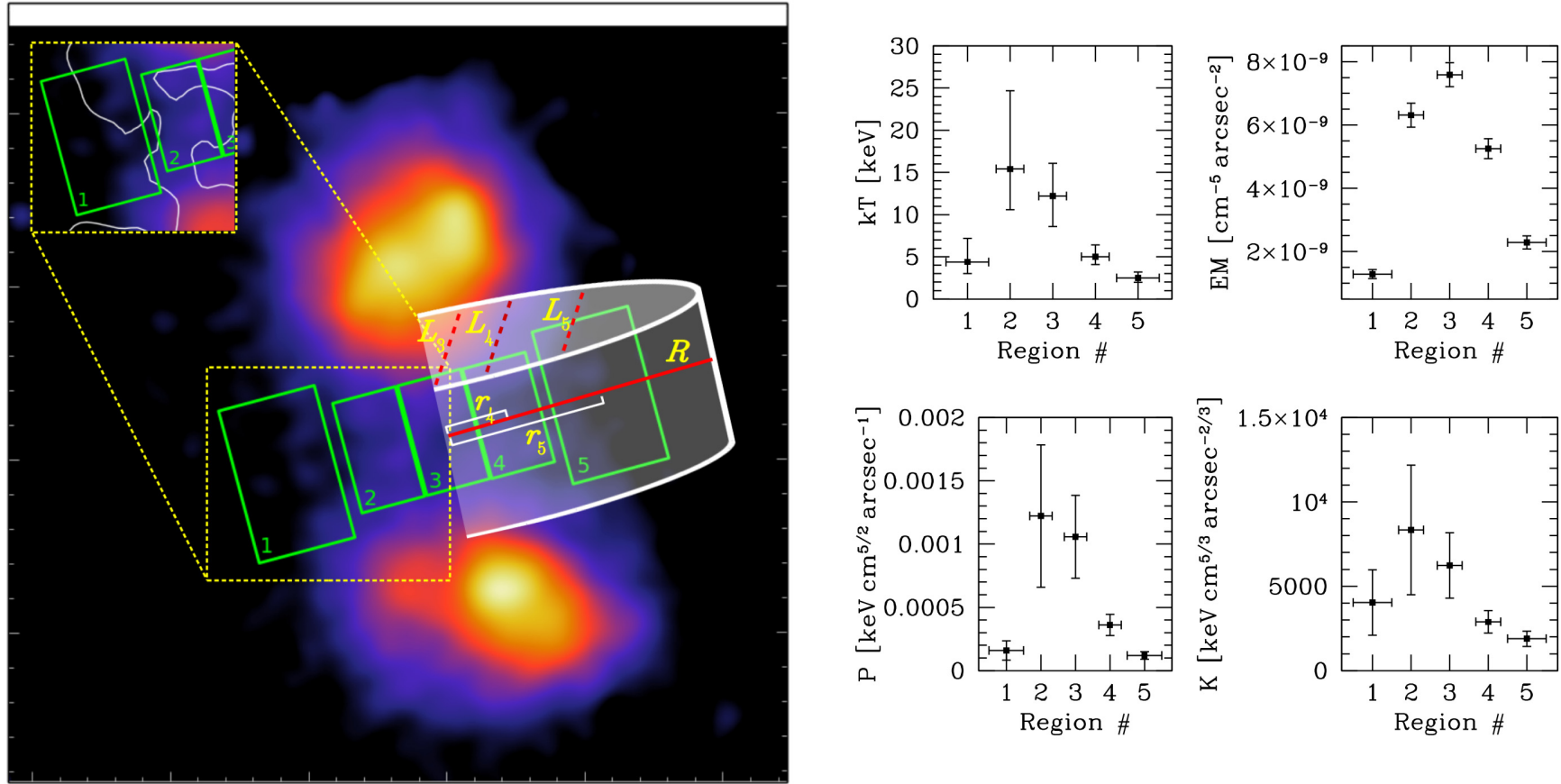

Figure 9. Left-hand panel: same Chandra image of Fig. 2 but with point sources subtracted and smoothed to a resolution of $\sim 15$ arcsec to highlight the $\mathrm{X}$-ray channel between the clusters. The spectral extracting regions are overlaid in green. The inset panel shows the spatial coincidence between the tentative radio bridge and the putative post-shock region. The cylindrical model assumed to assess the effects of projection is also sketched. The line of sight across the $i$-region is $L_{i} \sim 2 \sqrt{R^{2}-r_{i}^{2}}$. Right-hand panels: projected values of temperature $(k T)$, emission measure $(E M)$, pressure $(P)$, and entropy $(K)$ of the $\mathrm{X}$-ray channel.

a hint of SZ signal is also found between A1758N and A1758S (AMI Consortium 2012).

\subsection{The radio haloes in the A1758 complex}

It is known that giant radio haloes follow a relation between their radio power at $1.4 \mathrm{GHz}$ and the mass of the hosting cluster (e.g. Cassano et al. 2013). We used the values reported in Table 3 to calculate the $k$-corrected $1.4 \mathrm{GHz}$ radio power

$P_{1.4}=4 \pi S_{1.4} D_{L}^{2}(1+z)^{\alpha-1}$ for the two haloes in A1758N and A1758S, corresponding to $P_{1.4}^{\mathrm{N}}=$ $(6.3 \pm 0.4) \times 10^{24} \mathrm{~W} \mathrm{~Hz}^{-1}$ and $P_{1.4}^{\mathrm{S}}=(7.7 \pm 1.8) \times 10^{23} \mathrm{~W} \mathrm{~Hz}^{-1}$, respectively.

The $P_{1.4}-M_{500}$ relation reported in Cassano et al. (2013) was obtained using the masses derived from the Planck satellite via SZ effect, which is known to be a robust indicator of the cluster mass (e.g. Motl et al. 2005; Nagai 2006). However, the accuracy of the mass estimate for A1758N and A1758S with Planck is hindered by the difficulty of properly separating the two SZ components. Although the mass for A1758N has been estimated with different techniques, it is still uncertain and there is a large scatter in the values reported 


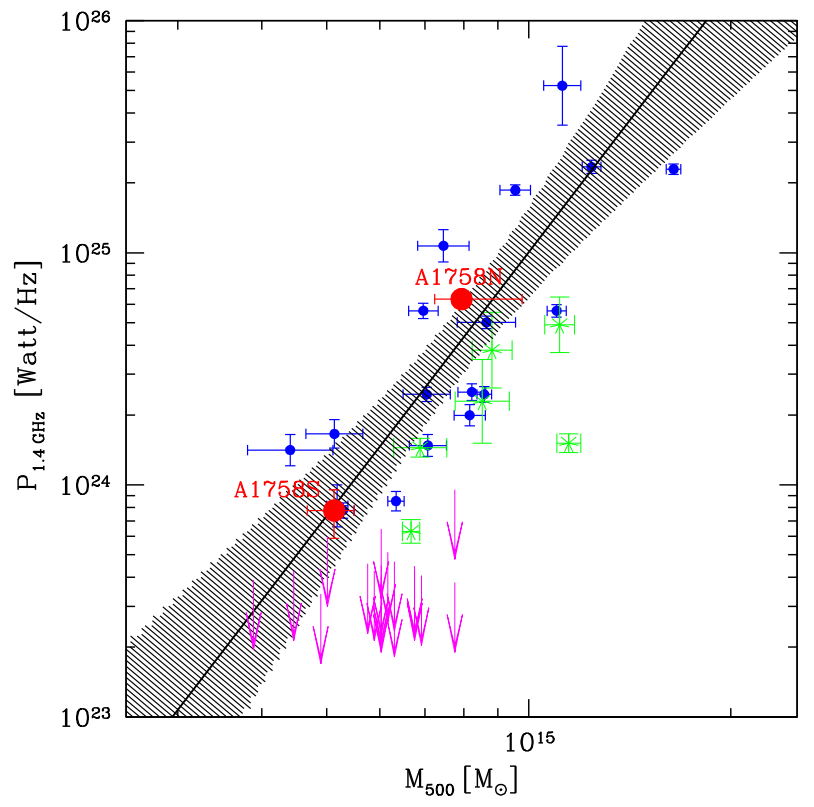

Figure 10. The $P_{1.4}-M_{500}$ relation for giant radio haloes. Different colours indicate: giant radio haloes (blue), ultrasteep spectrum radio haloes (green), upper limits from Venturi et al. (2008) (magenta), and the radio haloes in A1758N and A1758S (red). Errors on $P_{1.4}^{\mathrm{N}}$ are smaller than the point size. The best-fitting relation for giant radio haloes and its 95 per cent confidence level are shown. Adapted from Cassano et al. (2013).

in the literature (see table 1 in Monteiro-Oliveira et al. 2017). The mass of A1758S is even more uncertain due to the lack of literature studies focused on this sub-cluster. In this respect, we adopted the $M-T$ relation reported in Arnaud et al. (2005) to estimate $M_{500}$. We used the temperatures reported in David \& Kempner (2004), and derived $M_{500}^{\mathrm{N}}=8.0_{-0.8}^{+1.8} \times 10^{14} \mathrm{M}_{\odot}$ and $M_{500}^{\mathrm{S}}=(5.1 \pm 0.4) \times 10^{14}$ $\mathrm{M}_{\odot}$ for $\mathrm{A} 1758 \mathrm{~N}$ and $\mathrm{A} 1758 \mathrm{~S}$, respectively. We assumed these values being aware of the possible biases introduced in the scaling relation due to the ongoing mergers in A1758N and A1758S. However, we note that the masses estimated in such a way are within the values reported in the literature.

In Fig. 10 we compare our results with the $P_{1.4}-M_{500}$ relation reported in Cassano et al. (2013). The two radio haloes in the A1758 complex lie very close to the best-fitting curve. Our results are in agreement with the fact that the most powerful radio haloes are found in the most massive clusters.

\subsection{Merger scenario between A1758N and A1758S}

David \& Kempner (2004) did not find any excess emission in the $X M M-N e w t o n$ data in the region between A1758N and A1758S above that expected from a projection of the two systems. This suggested that the two components are not interacting because numerical simulations of merging clusters predict a surface brightness enhancement in the X-rays in the region of interaction (e.g. Roettiger et al. 1997; Ricker \& Sarazin 2001; Ritchie \& Thomas 2002). However, our observations provide new insights on the merger scenario between A1758N and A1758S.

Thanks to the new and deep Chandra observations we were able to produce maps of the ICM thermodynamical quantities of all the A1758 complex (Fig. 8). They highlight the presence of high temperature and high entropy plasma in the region between the clusters, suggesting the existence of shock heated gas. This has been observed in a number of binary X-ray clusters in an early merging phase (e.g. A1750, Belsole et al. 2004; A115, Gutierrez \& Krawczynski 2005; A3395, Lakhchaura et al. 2011; A98, Paterno-Mahler et al. 2014; CIZA J1358.9-4750, Kato et al. 2015; 1E2216.0-0401 and 1E2215.7-0404, Akamatsu et al. 2016; A399-A401, Akamatsu et al. 2017; A3653, Caglar \& Hudaverdi 2017; A141, Caglar 2018) and it is in agreement with predictions by numerical simulations (e.g. Takizawa 1999; Akahori \& Yoshikawa 2010). In contrast, the temperature enhancement is typically not observed when the separation of the pair exceeds their combined virial radii (e.g. A3528, Gastaldello et al. 2003; A3556-A3558, Mitsuishi et al. 2012; A3716, Andrade-Santos et al. 2015; A2467 Wegner et al. 2017). Further indication of compressed gas in A1758 is given by the transversal profiles of Fig. 9 that also pinpoint a drop of the computed quantities outside the X-ray channel, towards the east direction. We speculated that this region traces a transversal shock. Reasons for this include the high Mach number inferred from the temperature jump $\left(\mathcal{M}_{\mathrm{kT}}=3.0_{-1.0}^{+1.4}\right)$ and its position resembling that of the 'equatorial' shocks recently studied by Ha et al. (2018). These shocks are the first to form during the merger phase and have high velocities and high Mach numbers since they propagate in very low density regions, contrary to those found in between the cluster pairs that are weaker due to the high temperature of the central medium (e.g. Belsole et al. 2004; Paterno-Mahler et al. 2014; Kato et al. 2015; Akamatsu et al. 2016, 2017; Caglar 2018).

The $2 \sigma$ level radio emission connecting A1758N and A1758S observed with LOFAR (Fig. 4) needs further confirmation. If real, it could have been generated as a consequence of the encounter between the two clusters. This may indicate that part of the gravitational energy is dissipated into non-thermal components during the early phase of the merger. We find intriguing its co-location with the possible transversal shock suggested by the temperature profile in Fig. 9 (see also Fig. A2). A shock could indeed power the radio emission similarly to the case of radio relics, whilst equatorial shocks are less energetic due to the lower density of the upstream gas $^{6}$ (Ha et al. 2018).

In conclusion, the results coming from our radio/X-ray analysis are consistent with a scenario where A1758N and A1758S are in a pre-merger phase, where the clusters are approaching, the gas between them is compressed and heated and the first shocks are launched. The application of a two-body dynamical model (e.g. Beers et al. 1982) to test the gravitational binding of the clusters would be of great interest to probe the merging scenario; however, this is beyond the scope of this paper. Due to the overall complex dynamics of the merger (collision between clusters that are undergoing their own mergers), tailored numerical simulations would be useful to determine the impact velocities of the components in combination with multiwavelength data (see e.g. Molnar et al. 2013 for the A1750 case).

\section{CONCLUSIONS}

We have presented new LOFAR HBA observations of the double galaxy cluster A1758. In combination with archival VLA and GMRT data, we have constrained the spectral properties of the diffuse radio emission in the ICM. We also analysed a deep archival Chandra observation on the system. Here, we summarize our main results.

\footnotetext{
${ }^{6}$ The energy dissipated by shocks is $\propto n_{\mathrm{u}} V_{\text {sh }}^{3}$, where $n_{\mathrm{u}}$ is the upstream
} density and $V_{\mathrm{sh}}$ is the shock speed. 
(i) The radio halo in $\mathrm{A} 1758 \mathrm{~N}$ is well known in the literature. LOFAR has allowed us to recover diffuse radio emission from the ICM on a largest linear scale of $\sim 2.2 \mathrm{Mpc}$. The integrated spectral index computed from $144 \mathrm{MHz}$ to $1.4 \mathrm{GHz}$ is $\alpha=1.2 \pm 0.1$. The radio power of this halo is $P_{1.4}^{\mathrm{N}}=(6.3 \pm 0.4) \times 10^{24} \mathrm{~W} \mathrm{~Hz}^{-1}$.

(ii) Using LOFAR we have discovered a new, faint, radio halo in A1758S, which was not previously identified in studies at higher frequencies with the GMRT and VLA observations. Our reanalysis of these data sets revealed its elusive nature and constrained its spectral index between $144 \mathrm{MHz}$ and $1.4 \mathrm{GHz}$ to $\alpha=1.1 \pm 0.1$. The radio power of this halo is $P_{1.4}^{\mathrm{S}}=(7.7 \pm 1.8) \times 10^{23} \mathrm{~W} \mathrm{~Hz}^{-1}$.

(iii) Peripheral emission in the eastern outskirts of $\mathrm{A} 1758 \mathrm{~S}$ is also observed with LOFAR, GMRT, and VLA. We tentatively classified this source as a radio relic $(\alpha=1.3 \pm 0.1)$. Although the relic origin is suggested by a number of observational properties (e.g. morphology, location, linear extension), further observations are required to firmly determine its nature.

(iv) The two radio haloes in $\mathrm{A} 1758 \mathrm{~N}$ and $\mathrm{A} 1758 \mathrm{~S}$ lie within the 95 per cent confidence region of the best-fitting $P_{1.4}-M_{500}$ relation reported by Cassano et al. (2013).

(v) The maps of the ICM thermodynamical quantities computed from the deep Chandra observation indicate that the region between A $1758 \mathrm{~N}$ and A1758S is unrelaxed. In this respect, we suggested that the two sub-clusters are in a pre-merger phase.

(vi) A possible bridge of radio emission connecting A1758N and A1758S is suggested by the low-resolution LOFAR image. The ICM temperature across this bridge shows a drop possibly indicating the presence of a compressed region or a transversal shock generated in the initial stage of the merger that could play a role in the formation of this diffuse emission.

\section{ACKNOWLEDGEMENTS}

ABot thanks V. Cuciti for helpful advises during the radio data analysis, and F. Bedosti, T. J. Dijkema, and D. Rafferty for technical support on the LOFAR software installation. We thank F. Gastaldello, C. Haines, and F. Vazza for useful discussions, and the anonymous referee for suggestions that improved the manuscript. ABon acknowledges support from the ERC-Stg DRANOEL, no. 714245. RJvW, DNH, and HJAR acknowledge support from the ERC Advanced Investigator programme NewClusters 321271. RJvW acknowledges support from the VIDI research programme with project number 639.042.729, which is financed by the Netherlands Organization for Scientific Research (NWO). FdG is supported by the VENI research programme with project number 1808 , which is financed by the Netherlands Organization for Scientific Research (NWO). GJW gratefully acknowledges financial support from The Leverhulme Trust. This paper is based (in part) on data obtained with the International LOFAR Telescope (ILT) under project code LC2_038. LOFAR (van Haarlem et al. 2013) is the LOFAR designed and constructed by ASTRON. It has observing, data processing, and data storage facilities in several countries, that are owned by various parties (each with their own funding sources), and that are collectively operated by the ILT foundation under a joint scientific policy. The ILT resources have benefitted from the following recent major funding sources: CNRS-INSU, Observatoire de Paris and Université d'Orléans, France; BMBF, MIWF-NRW, MPG, Germany; Science Foundation Ireland (SFI), Department of Business, Enterprise and Innovation (DBEI), Ireland; NWO, The Netherlands; The Science and Technology Facilities Council, UK. This work had made use of the LOFAR Solution Tool (LoSoTo), developed by F. de Gasperin. We thank the staff of the GMRT that made these ob- servations possible. GMRT is run by the National Centre for Radio Astrophysics of the Tata Institute of Fundamental Research. The NRAO is a facility of the National Science Foundation operated under cooperative agreement by Associated Universities, Inc. The scientific results reported in this article are based on observations made by the Chandra X-ray Observatory. This research made use of APLpy, an open-source plotting package for PYTHON hosted at http://aplpy.github.com.

\section{REFERENCES}

Akahori T., Yoshikawa K., 2010, PASJ, 62, 335

Akamatsu H., Kawahara H., 2013, PASJ, 65, 16

Akamatsu H. et al., 2016, A\&A, 593, L7

Akamatsu H. et al., 2017, A\&A, 606, A1

AMI Consortium et al., 2012, MNRAS, 425, 162

Anders E., Grevesse N., 1989, Geochim. Cosmochim. Acta, 53, 197

Andrade-Santos F. et al., 2015, ApJ, 803, 108

Araya-Melo P., Aragón-Calvo M., Brüggen M., Hoeft M., 2012, MNRAS, 423, 2325

Arnaud K., 1996, in Jacoby G. H., Barnes J., eds., ASP Conf. Ser. Vol. 101, Astronomical Data Analysis Software and Systems V. Astron. Soc. Pac., San Francisco, p. 17

Arnaud M., Pointecouteau E., Pratt G., 2005, A\&A, 441, 893

Bartalucci I., Mazzotta P., Bourdin H., Vikhlinin A., 2014, A\&A, 566, A25

Beers T., Geller M., Huchra J., 1982, ApJ, 257, 23

Belsole E., Pratt G., Sauvageot J.-L., Bourdin H., 2004, A\&A, 415, 821

Bonafede A., Intema H., Brüggen M., Girardi M., Nonino M., Kantharia N., van Weeren R., Röttgering H., 2014, ApJ, 785, 1

Boschin W., Girardi M., Barrena R., Nonino M., 2012, A\&A, 540, A43

Botteon A., Gastaldello F., Brunetti G., Dallacasa D., 2016a, MNRAS, 460, L84

Botteon A., Gastaldello F., Brunetti G., Kale R., 2016b, MNRAS, 463, 1534

Botteon A., Gastaldello F., Brunetti G., 2018, MNRAS, 476, 5591

Brienza M. et al., 2016, A\&A, 585, A29

Briggs D., 1995, BAAS, 27, 1444

Brunetti G., 2016, Plasma Phys. Control. Fusion, 58, 14011

Brunetti G., Jones T., 2014, IJMPD, 23, 30007

Brunetti G., Lazarian A., 2011, MNRAS, 410, 127

Brunetti G., Lazarian A., 2016, MNRAS, 458, 2584

Brunetti G., Setti G., Feretti L., Giovannini G., 2001, MNRAS, 320, 365

Brunetti G., Venturi T., Dallacasa D., Cassano R., Dolag K., Giacintucci S., Setti G., 2007, ApJ, 670, L5

Brunetti G., Zimmer S., Zandanel F., 2017, MNRAS, 472, 1506

Buote D., 2001, ApJ, 553, L15

Caglar T., 2018, MNRAS, 475, 2870

Caglar T., Hudaverdi M., 2017, MNRAS, 472, 2633

Cash W., 1979, ApJ, 228, 939

Cassano R., Brunetti G., Röttgering H., Brüggen M., 2010a, A\&A, 509, A68

Cassano R., Ettori S., Giacintucci S., Brunetti G., Markevitch M., Venturi T., Gitti M., 2010b, ApJ, 721, L82

Cassano R. et al., 2013, ApJ, 777, 141

Chandra P., Ray A., Bhatnagar S., 2004, ApJ, 612, 974

Condon J., Cotton W., Greisen E., Yin Q., Perley R., Taylor G., Broderick J., 1998, AJ, 115, 1693

Cornwell T., Golap K., Bhatnagar S., 2005, in Shopbell P., Britton M., Ebert R., eds, ASP Conf. Ser. Vol. 347, Astronomical Data Analysis Software and Systems XIV. Astron. Soc. Pac., San Francisco, p. 86

Cuciti V., Cassano R., Brunetti G., Dallacasa D., Kale R., Ettori S., Venturi T., 2015, A\&A, 580, A97

Dahle H., Kaiser N., Irgens R., Lilje P., Maddox S., 2002, ApJS, 139, 313

David L., Kempner J., 2004, ApJ, 613, 831

de Gasperin F. et al., 2017, Sci. Adv., 3, e1701634

Drabent A., 2017, PhD thesis, Friedrich-Schiller-Universität Jena

Enßlin T., Biermann P., Klein U., Kohle S., Biermann P., Klein U., Kohle S., 1998, A\&A, 332, 395 
Feretti L., Giovannini G., Govoni F., Murgia M., 2012, A\&AR, 20, 54

Gastaldello F., Ettori S., Molendi S., Bardelli S., Venturi T., Zucca E., 2003, A\&A, 411, 21

Giovannini G., Bonafede A., Feretti L., Govoni F., Murgia M., Ferrari F., Monti G., 2009, A\&A, 507, 1257

Guo X., Sironi L., Narayan R., 2014a, ApJ, 794, 153

Guo X., Sironi L., Narayan R., 2014b, ApJ, 797, 47

Gutierrez K., Krawczynski H., 2005, ApJ, 619, 161

Ha J.-H., Ryu D., Kang H., 2018, ApJ, 857, 26

Haines C., Smith G., Egami E., Okabe N., Takada M., Ellis R., Moran S., Umetsu K., 2009, MNRAS, 396, 1297

Haines C. et al., 2017, preprint (arXiv:1709.04945)

Hardcastle M. et al., 2016, MNRAS, 462, 1910

Hoang D. et al., 2017, MNRAS, 471, 1107

Hoeft M., Brüggen M., 2007, MNRAS, 375, 77

Intema H., van der Tol S., Cotton W., Cohen A., van Bemmel I., Röttgering H., 2009, A\&A, 501, 1185

Intema H., Jagannathan P., Mooley K., Frail D., 2017, A\&A, 598, A78

Kalberla P., Burton W., Hartmann D., Arnal E., Bajaja E., Morras R., Pöppel W., 2005, A\&A, 440, 775

Kang H., Petrosian V., Ryu D., Jones T., 2014, ApJ, 788, 142

Kang H., Ryu D., 2011, ApJ, 734, 18

Kang H., Ryu D., 2016, ApJ, 823, 13

Kang H., Ryu D., Jones T., 2012, ApJ, 756, 97

Kato Y., Nakazawa K., Gu L., Akahori T., Takizawa M., Fujita Y., Makishima K., 2015, PASJ, 67, 71

Kempner J., Sarazin C., 2001, ApJ, 548, 639

Keshet U., Waxman E., Loeb A., 2004, ApJ, 617, 281

Lakhchaura K., Singh K., Saikia D., Hunstead R., 2011, ApJ, 743, 78

Landau L., Lifshitz E., 1959, Course of Theoretical Physics. Pergamon Press, Oxford

Lane W., Cotton W., van Velzen S., Clarke T., Kassim N., Helmboldt J., Lazio T., Cohen A., 2014, MNRAS, 440, 327

Macario G., Markevitch M., Giacintucci S., Brunetti G., Venturi T., Murray S., 2011, ApJ, 728, 82

Machado R., Monteiro-Oliveira R., Lima Neto G., Cypriano E., 2015, MNRAS, 451, 3309

Markevitch M., Vikhlinin A., 2007, Phys. Rep., 443, 1

Martino R., Mazzotta P., Bourdin H., Smith G., Bartalucci I., Marrone D., Finoguenov A., Okabe N., 2014, MNRAS, 443, 2342

Mazzotta P., Rasia E., Moscardini L., Tormen G., 2004, MNRAS, 354, 10

McMullin J., Waters B., Schiebel D., Young W., Golap K., 2007, in Shaw R., Hill F., Bell D., eds., ASP Conf. Ser. Vol. 376, Astronomical Data Analysis Software and Systems XVI. Astron. Soc. Pac., San Francisco, p. 127

Mitsuishi I. et al., 2012, PASJ, 64, 18

Mohan N., Rafferty D., 2015, Astrophysics Source Code Library, ascl: 1502.007

Molnar S., Chiu I.-N., Broadhurst T., Stadel J., 2013, ApJ, 779, 63

Monteiro-Oliveira R., Cypriano E., Machado R., Lima Neto G., Ribeiro A., Sodré L., Dupke R., 2017, MNRAS, 466, 2614

Motl P., Hallman E., Burns J., Norman M., 2005, ApJ, 623, L63

Murgia M., Govoni F., Feretti L., Giovannini G., 2010, A\&A, 509, A86

Nagai D., 2006, ApJ, 650, 538
Nuza S., Hoeft M., van Weeren R., Gottlöber S., Yepes G., 2012, MNRAS, 420, 2006

O’Dea C., Owen F., 1985, AJ, 90, 927

Offringa A. et al., 2014, MNRAS, 444, 606

Okabe N., Umetsu K., 2008, PASJ, 60, 345

Paterno-Mahler R., Randall S., Bulbul E., Andrade-Santos F., Blanton E., Jones C., Murray S., Johnson R., 2014, ApJ, 791, 104

Perley R., Butler B., 2013, ApJS, 204, 19

Petrosian V., 2001, ApJ, 557, 560

Pinzke A., Oh S., Pfrommer C., 2013, MNRAS, 435, 1061

Pinzke A., Oh S., Pfrommer C., 2017, MNRAS, 465, 4800

Planck Collaboration VIII, 2013, A\&A, 550, A134

Ragozzine B., Clowe D., Markevitch M., Gonzalez A., Bradač M., 2012, ApJ, 744, 94

Rau U., Cornwell T., 2011, A\&A, 532, A71

Rengelink R., Tang Y., de Bruyn A., Miley G., Bremer M., Roettgering H., Bremer M., 1997, A\&AS, 124

Ricker P., Sarazin C., 2001, ApJ, 561, 621

Ritchie B., Thomas P., 2002, MNRAS, 329, 675

Rizza E., Burns J., Ledlow M., Owen F., Voges W., Bliton M., 1998, MNRAS, 301, 328

Roettiger K., Burns J., Stone J., 1999, ApJ, 518, 603

Roettiger K., Loken C., Burns J., 1997, A\&AS, 109, 307

Röttgering H. et al., 2011, J. Astrophys. Astron., 32, 557

Röttgering H. et al., 2006, preprint (astro-ph/0610596)

Sanders J., 2006, MNRAS, 371, 829

Savini F. et al., 2018, MNRAS, 474, 5023

Scaife A., Heald G., 2012, MNRAS, 423, L30

Shimwell T., Markevitch M., Brown S., Feretti L., Gaensler B., JohnstonHollitt M., Lage C., Srinivasan R., 2015, MNRAS, 449, 1486

Shimwell T. et al., 2016, MNRAS, 459, 277

Shimwell T. et al., 2017, A\&A, 598, A104

Sirothia S., 2009, MNRAS, 398, 853

Takizawa M., 1999, ApJ, 520, 514

Tasse C., van der Tol S., van Zwieten J., van Diepen G., Bhatnagar S., 2013, A\&A, 553, A105

van Haarlem M. et al., 2013, A\&A, 556, A2

van Weeren R. et al., 2016a, ApJ, 818, 204

van Weeren R. et al., 2016b, ApJS, 223, 2

van Weeren R. et al., 2017, Nat. Astron., 1, 5

Vazza F., Brüggen M., 2014, MNRAS, 437, 2291

Vazza F., Eckert D., Brüggen M., Huber B., 2015a, MNRAS, 451, 2198

Vazza F., Ferrari C., Brüggen M., Bonafede A., Gheller C., Wang P., 2015b, A\&A, 580, A119

Vazza F., Brüggen M., Wittor D., Gheller C., Eckert D., Stubbe M., 2016, MNRAS, 459, 70

Venturi T., Giacintucci S., Dallacasa D., Cassano R., Brunetti G., Bardelli S., Setti G., 2008, A\&A, 484, 327

Venturi T., Giacintucci S., Dallacasa D., Cassano R., Brunetti G., Macario G., Athreya R., 2013, A\&A, 551, A24

Wegner G. et al., 2017, ApJ, 844, 67

Wilber A. et al., 2018, MNRAS, 473, 3536

Williams W. et al., 2016, MNRAS, 460, 2385

Wittor D., Vazza F., Brüggen M., 2017, MNRAS, 464, 4448 
APPENDIX A: TEMPERATURE MAP

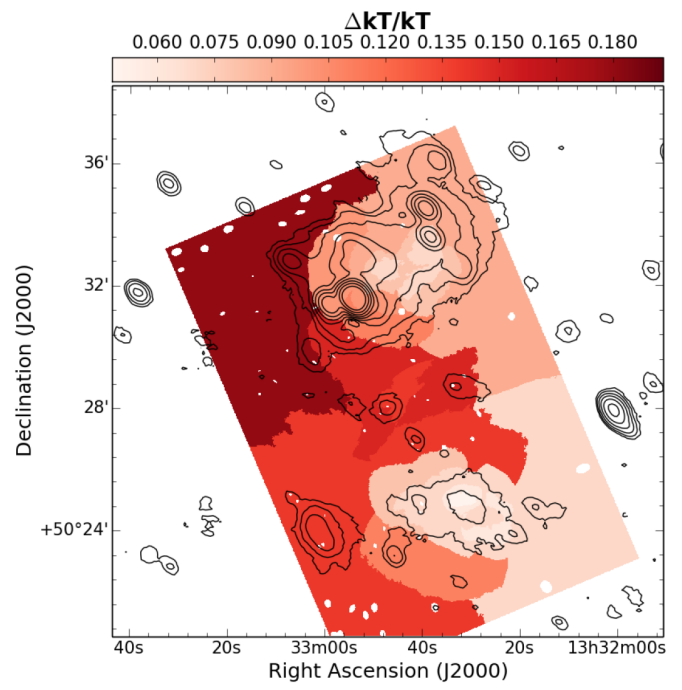

Figure A1. Temperature error map of A1758 (cf. with Fig. 8).

The fractional errors on the temperature map of Fig. 8 are reported in Fig. A1. Projected pressure and entropy have similar fractional errors due to their linear dependence on the temperature and to the general small error on the emission measure.
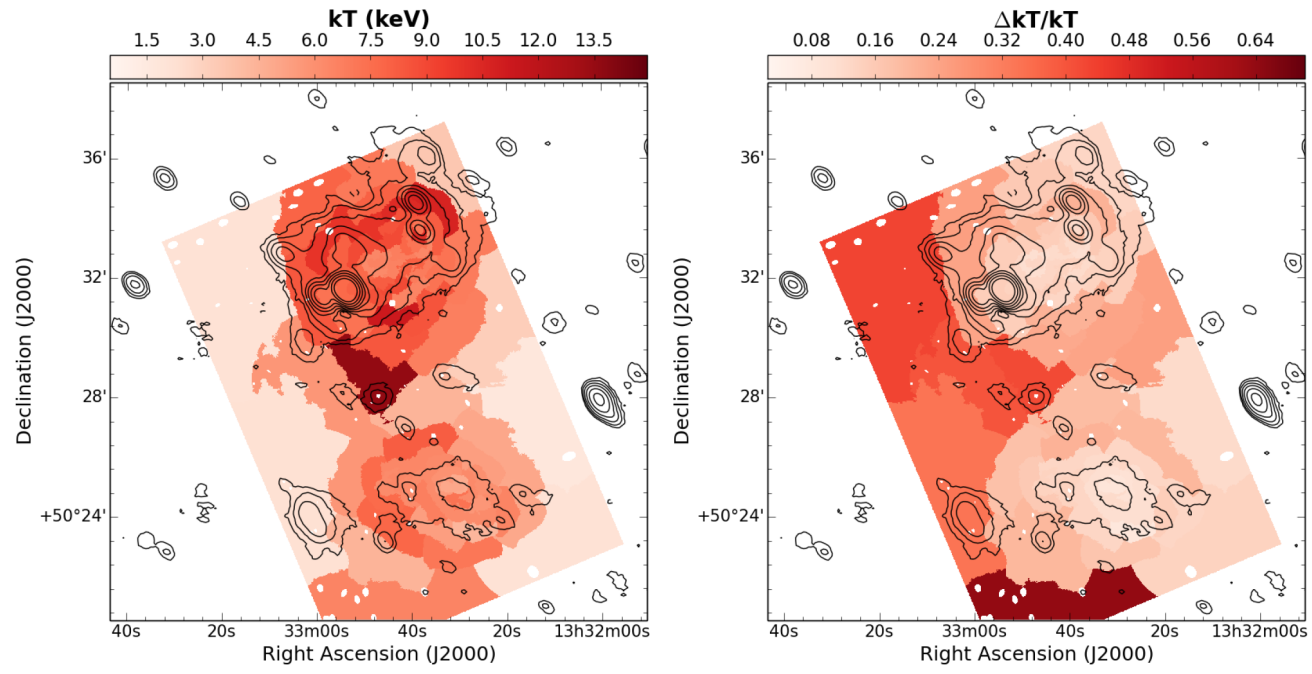

Figure A2. Low S/N temperature map (left) and relative error map (right) of A1758.

The high value of temperature in the putative post-shock region of Fig. 9 cannot be identified in the temperature map of Fig. 8 (left-hand panel) likely due to the fact that the spectral extracting sectors are large, hence they might contain a mix of plasma with different temperatures. In Fig. A2 we show that if the required S/N per region is reduced to 30, the ConTBIN algorithm is able to draw a smaller sector similar in size and position to region 2 in Fig. 9 where the spectral fit provides again $k T \sim 15 \mathrm{keV}$, cancelling the apparent tension between the two results.

\section{APPENDIX B: X-RAY CHANNEL SPECTRA}

The best-fitting spectra of the five regions shown in Fig. 9 are reported in Fig. B1. The spectral model components are depicted with different colours in the plots. To assess the impact of the systematic uncertainties of the background modelling to the estimates of the ICM temperature, we re-performed the spectral fits varying within $\pm 1 \sigma$ the normalization levels first of the instrumental background alone, and later of both the instrumental and astrophysical backgrounds. The results are summarized in Table B1 and are consistent within $1 \sigma$ with that reported in Fig. 9 (right-hand panel). Finally, we mention that the drop of the Chandra effective area above $5 \mathrm{keV}$ makes the estimation of high temperatures critical with this instrument. In this respect, the errors on the high temperatures reported in Table B1 may not reflect entirely the real range of statistical and systematic uncertainties. 

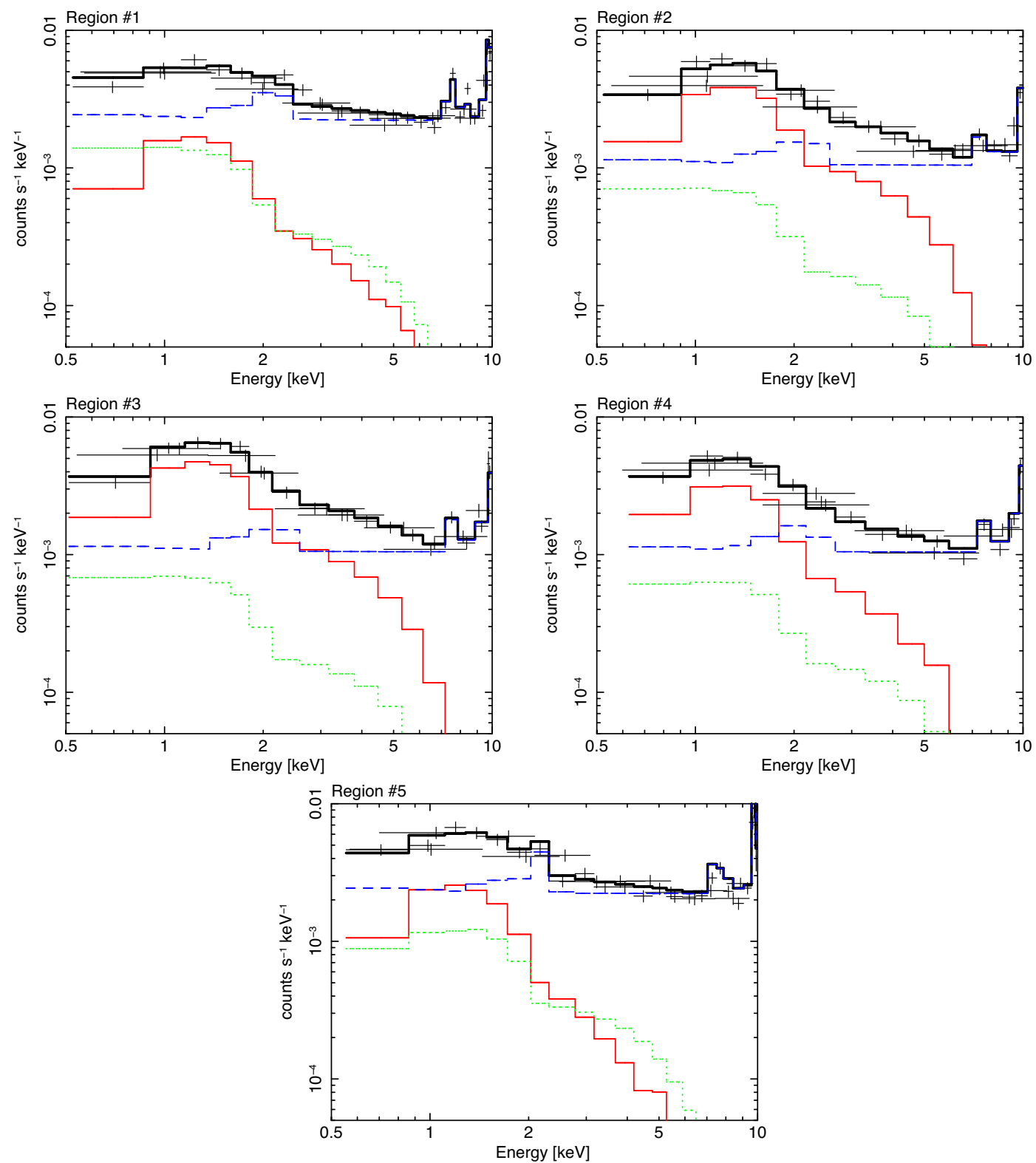

Figure B1. Spectral fitting results for the five regions shown in Fig. 9 (left-hand panel). Data points are shown in black together with the best-fitting model. Different colours denote the components of the spectral model; i.e. the cluster emission (in solid red), the astrophysical background (in dotted green) and the instrumental background (in dashed blue). Whilst the three ObsID spectra were simultaneously fitted, the models for only one observation were reported in order to avoid confusion in the plot. The c-stat/d.o.f. of the fits from region 1 to 5 are: 232/184, 135/127, 114/132, 115/113, and 224/182.

Table B1. Impact of the systematic uncertainties of the background modelling on the temperature estimates reported in Fig. 9 (right-hand panel). Tests were performed varying within $\pm 1 \sigma$ the normalization level of the instrumental background ('NXB') and of the astrophysical background ('sky'). Temperatures are reported in $\mathrm{keV}$ units.

\begin{tabular}{|c|c|c|c|c|c|}
\hline \multirow[t]{2}{*}{ Region } & \multirow[t]{2}{*}{ Best fit } & \multicolumn{2}{|c|}{ NXB } & \multicolumn{2}{|c|}{ NXB + Sky } \\
\hline & & $+1 \sigma$ & $-1 \sigma$ & $+1 \sigma$ & $-1 \sigma$ \\
\hline 1 & $4.4_{-1.4}^{+2.8}$ & $3.7_{-1.0}^{+2.1}$ & $5.4_{-1.9}^{+4.1}$ & $3.5_{-1.5}^{+4.1}$ & $5.1_{-1.1}^{+3.4}$ \\
\hline 3 & $12.2_{-3.6}^{+3.9}$ & $10.2_{-2.3}^{+4.5}$ & $13.6_{-3.9}^{+3.5}$ & $11.8_{-3.4}^{+4.1}$ & $13.2_{-3.7}^{+3.5}$ \\
\hline 4 & $5.0_{-0.9}^{+1.4}$ & $4.5_{-0.7}^{+1.2}$ & $5.4_{-1.1}^{+1.5}$ & $4.8_{-0.9}^{+1.4}$ & $5.3_{-1.0}^{+1.5}$ \\
\hline 5 & $2.5_{-0.5}^{+0.5}$ & $2.2_{-0.3}^{+0.6}$ & $2.7_{-0.5}^{+0.9}$ & $2.3_{-0.4}^{+0.5}$ & $2.8_{-0.6}^{+0.8}$ \\
\hline
\end{tabular}

This paper has been typeset from a $\mathrm{T}_{\mathrm{E}} \mathrm{X} / \mathrm{L} \mathrm{T}_{\mathrm{E}} \mathrm{X}$ file prepared by the author. 